\title{
Depression is closely associated with discoordination between the heart rate variability and physical acceleration in free-moving older women
}

Kentaro Taniguchi

Kyoto Daigaku

Akito Shimouchi ( $\square$ ashimouc@isc.chubu.ac.jp )

Chubu Daigaku https://orcid.org/0000-0002-2623-381X

Naoya Jinno

Chubu Daigaku

Akitoshi Seiyama

Kyoto Daigaku

Research article

Keywords: Heart Rate Variability, Physical Acceleration, Depression, Cross-correlation

Posted Date: July 22nd, 2020

DOI: https://doi.org/10.21203/rs.3.rs-36613/v1

License: (c) (1) This work is licensed under a Creative Commons Attribution 4.0 International License.

Read Full License 


\section{Abstract \\ Background}

We investigated the relationship between psychological distress and the coordination of heart rate variability (HRV) and physical acceleration (PA) during free-moving days in women.

\section{Methods}

Ninety-five adult women were divided into younger and older groups. The younger group comprised 50 women (22-59 years), and the older group comprised 45 women ( $\geq 60$ years). HRV and PA data were simultaneously obtained every minute for $24 \mathrm{~h}$ during the free-moving day. The ratios of low frequency/high frequency and high frequency in normalized units were used as HRV indices. We defined $\%$ Lag0 as the \% frequency of the lag $=0$ min between HRV and PA in $1 \mathrm{~h}$. The General Health Questionnaire 28 (GHQ28) was used to evaluate the effects of psychological distress and depression.

\section{Results}

In the hour before the night's sleep, \%Lag0 was significantly lower in older women with depression (GHQ28 subscale D) than in older women without depression $(p<0.05)$. However, no significant difference between \%Lag0 and depression status was observed in the hour after waking in older women.

\section{Conclusions}

The results suggest that impairments in coordination between HRV and PA are associated with depression in older women, particularly in the hour before a night's sleep on free-moving days.

\section{Background}

Heart rate variability (HRV) is a non-invasive tool for the assessment of variations in beat-to-beat intervals and autonomic nervous system activity. Most human studies on the HRV spectrum have examined the power of high frequency (HF) and low frequency (LF) ranges [1, 2]. Normalized spectral indices, defined as $\mathrm{HFnu}=\mathrm{HF} /(\mathrm{LF}+\mathrm{HF})$, are regarded as markers of the parasympathetic nervous system [3]. It has also been reported that increases in LF/HF may represent a shift towards sympathetic predominance and reduced vagal activity [4, 5]; thus, it has been used as a measure of sympathovagal balance [6], although the physiological underpinnings of this metric are controversial [7].

In contrast, physical acceleration (PA) is utilized to evaluate physical activity in subjects with severe mental illness [8] and in several clinical settings [9]. During exercise with an increasing PA, in addition to an increase in heart rate, an increase in sympathetic activity and a decrease in vagal discharge are 
detected [10]. Moreover, previous studies have shown that immediately after the initiation of exercise, heart rate increases due to a withdrawal of parasympathetic nervous activity and an increase in sympathetic nervous activity [11]. Under healthy conditions, we considered that PA causes an immediate HRV response, whereas in impaired conditions the response would be delayed. Therefore, we considered that this lag may indicate the level of coordination between PA and neuro-cardiovascular systems including the autonomic nervous system. If the coordination is impaired, we considered that the harmony between the autonomic nervous system and PA may be disrupted. We considered that the lag between PA and HRV was affected not only by direct interaction between the autonomic nervous system and physical activity, but also by the higher central nervous system including mental or psychological conditions. To investigate the association between HRV and a physiological signal, cross-correlation analysis is widely used [12]. Based on a cross-correlation analysis between the time series of HRV and PA, we reported that an increase in the lag time (i.e. \%Lag0 defined as the percent ratio of the lag $=0 \mathrm{~min}$ in $1 \mathrm{~h}$ ) decreases with aging in the daily lives of free-moving adults [13]. We also found that the most appropriate time to detect impairments in coordination between HRV and PA is in the hour before a night's sleep.

Plausible mechanisms to explain the association between PA and depression include PA-induced changes in physiological/neurological and psychological parameters [14]. PA may prevent depression by increasing the functional activity of monoamines [15]. We previously reported that \%Lag0 decrease was closely associated with psychological stress [13]. However, a sex dependency remained unclear, most likely due to differences in the hormonal milieu and brain structure between sexes. Moreover, depression has sex-specific phenotypes [16]. Since habitual activity and HRV have both been reported to differ between men and women $[17,18]$, the present study focused on adult women.

It is reported that active and passive changes in fundamentally different cardiovascular effects for approximately 20 seconds involve the central command, muscle receptors, high-pressure receptors, and low-pressure receptors [19]. Moreover, in a head-up tilt test, a gradual change in hemodynamic parameters is observed, with plateau levels being reached in 20-30 seconds [6]. Therefore, it is considered that the lag between PA and hemodynamic parameters would express the coordination levels in neuro-cardiovascular systems.

We investigated whether psychological distress is associated with impairments in coordination between HRV and PA in daily life during free-moving days in adult women. Our investigation combined \%Lag0 data and the General Health Questionnaire 28 (GHQ28), which is frequently used as an indicator of psychological distress $[20,21]$

\section{Methods}

\section{Participants}

One hundred and six volunteers participated in this study. Study participants were adult women whose daily activities were not restricted by disease or injury. Participants that were excluded included 5 who 
drank alcohol on the experimental day, 6 with significant arrhythmia, 2 taking beta-blockers, and 3 with excessive electrical noise in the PA devices described below (these numbers overlapped). All participants were screened based on their responses to medical interviews about their previous and present illnesses, physical findings, expired gas screening, blood test results, and ECG (Fig. 1). Arrhythmia was also identified based on the assessment of a 12-lead ECG at rest, and Lorenz plots [22] obtained using electrocardiographic complexes for 24 hours. Ninety-five participants aged between 22 and 85 years were included in this study. Of these, 4 had type II diabetes, 17 had hypertension, 35 had dyslipidaemia, 6 had hepatic dysfunction, and 2 had renal dysfunction. However, none of the participants had restricted movement.

\section{Protocols}

Participants completed GHQ28 questionnaires before or on the day of the assessment and were then brought to the laboratory. They arrived at the laboratory at approximately 13:00 and underwent a physical examination and ECG. The participants then wore a portable monitor (Active Tracer AC301; GMS Inc., Tokyo, Japan) to record PA and R-R intervals for 24 hours. During monitoring, participants were instructed to continue with their normal lives, but to avoid bathing. After the completion of the 24 hour monitoring period, each participant returned to the laboratory. Experimental protocols were described in detail in our previous studies $[13,23]$.

\section{Questionnaires}

We used the Japanese version of GHQ28 to evaluate mental or psychological distress (Nihon Bunka Kagakusya Co., Ltd.). GHQ28 is a self-report instrument and is frequently used as an indicator of psychological well-being and the psychological dimensions of quality of life [20]. GHQ28 has 4 subscales (A: somatic symptoms; B: anxiety and insomnia; C: social impairments; D: severe depression) [20]. Each of these subscales includes 7 items. Items were scored using 2-point scores of 0-0-1-1, and the sum of these scores reportedly indicates the severity of mental or psychological distress [20]. GHQ scores were defined as low (total $<8, A$ and $B<2, C$ and $D=0$ ) and high (total $\geq 8, A$ and $B \geq 2, C$ and $D \geq 1$ ) [24].

\section{Physical acceleration (PA)}

To measure PA, an Active Tracer equipped with a triaxial accelerometer (72 $\mathrm{g}$ in weight) was used [25]. The body of the accelerometer was positioned on the frontal midline of the waist above the navel to avoid disturbance of sleep or free movement. The resolution of acceleration was $2 \mathrm{mG}$, and the sensitivity ranged between 0 and $4.0 \mathrm{G}$. The absolute values of the resultant vector, which were calculated from the signals of triaxial acceleration, were averaged for every minute. The times at which participants fell asleep and woke up were estimated based on the records kept by the participants and changes in body positions evaluated from the acceleration vector. We defined three periods as follows: evening - from the start of the monitoring to sleep at night; sleep: subjects on the bed; morning - after waking up to the end of the monitoring.

\section{Participants in each group}


A previous report showed that older adults (aged more than 60 years) in Japan were better protected from distress from a disaster compared with younger adults. Depression was also different from those groups [26]. Therefore, in this study, we divided the participants into younger and older age groups based on a cut-off of 60 years old.

Ninety-five subjects were divided into 4 groups: younger with low distress (age $<60$ years and GHQ total score $<8, n=37, I$ ), younger with high distress (age $<60$ years and GHQ total score $\geq 8, n=13$, II), older with low distress (age $\geq 60$ years and $G H Q$ total score $<8, n=26$, III), and older with high distress (age $\geq$ 60 years and GHQ total score $\geq 8, n=19, I V)$.

\section{Analysis of HRV}

The time-domain analysis and spectral analysis of HRV were performed at 1-minute intervals using maximal entropy combined with the least square method (MEMCalc System; Suwa Trust Co., Ltd., Tokyo). After power spectra density estimation, we separated the signals into the HF range $(0.15-0.4 \mathrm{~Hz})$ and LF range $(0.04-0.15 \mathrm{~Hz})$ for power analysis [1]. In this study, we defined HRV indices as HFnu = $\mathrm{HF} /(\mathrm{LF}+\mathrm{HF})$ and LF/HF, which have been regarded as markers of the autonomic nervous system [1]. HFnu is generally associated with cardiac vagal balance [2]. Increased LF/HF may represent a shift towards sympathetic predominance and reduced vagal activity [5].

\section{Definition of \%Lag0}

Cross-correlation is a mathematical calculation that represents the similarity of two signals changing over time, allowing for a defined lag time. Lag time was determined as the time difference indicated by the maximum absolute cross-correlation coefficients obtained from an analysis between the HRV components (HFnu or LF/HF) and the PA. Cross-correlation coefficients were calculated for 10-min time windows over consecutive 60-min periods. In our preliminary cross-correlation analysis, we confirmed that similar results were obtained regardless of whether we used a time window of 10 or 20 min for evaluating the lag between HRV and PA.

In previous cross-correlation studies, HRV components were extracted every 5 min [27]. However, the coordination between physical activity and the autonomic nervous system is generally considered to involve a short time frame under normal physiological conditions. Thus, we employed 1 min data collection intervals to obtain more precise information. Then, we defined impairments of coordination between HRV and PA when the lag time was 1 min or longer. We then defined the \%Lag0 as the percent ratio of the lag $=0$ min in $1 \mathrm{~h}$ and an index of coordination between HRV and PA. Thus, low levels of \%Lag0 would indicate impairment of coordination between PA and HRV.

Typically, different social events happen in the daytime period for most people, during which \%Lag0 could vary markedly depending upon the participants. Moreover, \%Lag0 between PA and HF or LF/HF before sleep (evening) was slightly higher than that after waking up (morning) among the older and younger groups (data for reviewers). Therefore we considered that both the hour before sleeping and that after 
waking appeared to involve less social activities, and restricted the analysis of \%Lag0 to the hour before sleeping and after waking up.

\section{Statistical analysis}

Data are expressed as the mean \pm standard error of the mean. Statistical analysis was appropriately performed with one-way analysis of variance followed by Tukey's test, and an unpaired t-test using Excel and SPSS. All $p<0.05$ were considered statistically significant.

\section{Ethics}

This study was approved by the Ethics Committee at the National Cerebral and Cardiovascular Research Center (M18-19-2, M26-158), Chubu University (280031) and Kyoto University (R1758). All participants provided written informed consents prior to participation in this study.

\section{Results}

We defined sleep time as the time participants were in bed, evening from the start of monitoring to falling asleep at night, and morning from after waking up to the end of the analysis. As shown in Table 1 no significant differences were observed in basic characteristics (age, body mass index, and sleeping hours), PA, and HRV (LF/HF and HFnu), except for PA in the evening, between the high- and low-distress groups of the same age group. In the older group, PA in the evening was significantly lower in the high-distress group (IV) than that in the low-distress group (III). During their night's sleep, all participants showed significantly lower LF/HF and higher HFnu than during waking time. Among older subjects, the highdistress group (IV) had significantly higher frequencies of nocturnal awakening than the low-distress group (III). 
Table 1

Comparisons of demographic, questionnaire, and extracted parameters between General Health Questionnaire 28 (GHQ28) total scores

\begin{tabular}{|c|c|c|c|c|c|}
\hline \multicolumn{2}{|l|}{ Group } & I & II & III & IV \\
\hline \multicolumn{2}{|c|}{ Age (years) } & $<60$ & $<60$ & $\geq 60$ & $\geq 60$ \\
\hline \multicolumn{2}{|c|}{ GHQ total score } & $\begin{array}{l}\text { GHQ-score }< \\
8\end{array}$ & $\begin{array}{l}\text { GHQ-score } \geq \\
8\end{array}$ & $\begin{array}{l}\text { GHQ-score }< \\
8\end{array}$ & $\begin{array}{l}\text { GHQ-score } \geq \\
8\end{array}$ \\
\hline \multicolumn{2}{|l|}{$N$} & 37 & 13 & 26 & 19 \\
\hline \multicolumn{2}{|c|}{ Age (years) } & $42.8 \pm 1.9$ & $42.2 \pm 3.6$ & $69.8 \pm 1.1$ & $71.4 \pm 1.4$ \\
\hline \multicolumn{2}{|c|}{ Body mass index } & $21.6 \pm 0.4$ & $23.2 \pm 0.8$ & $23.1 \pm 0.7$ & $22.4 \pm 0.6$ \\
\hline \multicolumn{2}{|c|}{ Sleeping hours } & $6.8 \pm 0.2$ & $7.1 \pm 0.5$ & $7.2 \pm 0.3$ & $7.5 \pm 0.3$ \\
\hline \multicolumn{2}{|c|}{$\begin{array}{l}\text { Frequency of nocturnal } \\
\text { awakening }\end{array}$} & $0.46 \pm 0.12$ & $0.54 \pm 0.22$ & $0.73 \pm 0.15$ & $1.41 \pm 0.22^{*}$ \\
\hline \multicolumn{2}{|c|}{ GHQ A: somatic symptoms } & $1.2 \pm 0.2$ & $4.1 \pm 0.4^{\#}$ & $1.4 \pm 0.3$ & $3.3 \pm 0.5^{\star}$ \\
\hline \multicolumn{2}{|c|}{ GHQ B: anxiety \& insomnia } & $1.5 \pm 0.2$ & $4.5 \pm 0.5^{\#}$ & $1.4 \pm 0.3$ & $5.4 \pm 0.4^{*}$ \\
\hline \multicolumn{2}{|c|}{ GHQ C: social impairment } & $0.2 \pm 0.1$ & $2.2 \pm 0.6^{\#}$ & $1.1 \pm 0.1$ & $2.6 \pm 0.4^{*}$ \\
\hline \multicolumn{2}{|c|}{ GHQ D: severe depression } & $0.1 \pm 0.0$ & $1.8 \pm 0.6^{\#}$ & $0.0 \pm 0.0$ & $2.7 \pm 0.6^{*}$ \\
\hline \multirow[t]{2}{*}{$\mathrm{PA}(\mathrm{mG})$} & evening & $37.3 \pm 1.9$ & $44.0 \pm 5.4$ & $44.6 \pm 2.2$ & $33.8 \pm 2.7^{*}$ \\
\hline & morning & $49.8 \pm 3.1$ & $51.3 \pm 5.2$ & $54.8 \pm 4.8$ & $44.9 \pm 3.3$ \\
\hline \multirow[t]{3}{*}{ HFnu } & evening & $0.26 \pm 0.01$ & $0.27 \pm 0.02$ & $0.28 \pm 0.02$ & $0.32 \pm 0.02$ \\
\hline & sleep & $0.46 \pm 0.02$ & $0.45 \pm 0.04$ & $0.40 \pm 0.03$ & $0.41 \pm 0.03$ \\
\hline & morning & $0.24 \pm 0.01$ & $0.26 \pm 0.02$ & $0.29 \pm 0.02$ & $0.31 \pm 0.02$ \\
\hline \multirow[t]{3}{*}{ LF/HF } & evening & $6.06 \pm 0.44$ & $5.40 \pm 0.75$ & $4.97 \pm 0.41$ & $4.43 \pm 0.44$ \\
\hline & sleep & $2.61 \pm 0.23$ & $2.66 \pm 0.47$ & $3.56 \pm 0.42$ & $3.48 \pm 0.44$ \\
\hline & morning & $7.01 \pm 0.51$ & $5.74 \pm 0.68$ & $4.79 \pm 0.43$ & $4.57 \pm 0.41$ \\
\hline \multicolumn{6}{|l|}{. } \\
\hline \multicolumn{6}{|c|}{$\begin{array}{l}\#: p<0.05 \text { I vs. II, *: } p<0.05 \text { III vs. IV Evening: from the start of the monitoring to sleep at night; } \\
\text { morning: after waking up to the end of the monitoring, and night time: subjects on the bed. }\end{array}$} \\
\hline
\end{tabular}

Scores for each GHQ subscale were significantly higher in the younger and older high-distress groups (II and IV) than those in the younger and older low-distress groups (I and III), respectively. 
Data from a subject with low depression with lag $=0$ of PA and LF/HF before sleep are shown in Fig. 2 as an example. Data from a subject with high depression with a lag $=2 \mathrm{~min}$ of PA and LF/HF before sleep are shown in Fig. 3 as an example. This data indicates that PA preceded any change in LF/HF.

Relationships between age and \%Lag0 in the hour before and after sleep are shown in Table 2. The \%Lag0 between HRV parameters and PA was significantly lower in the older group than in the younger group in the hour before and after sleep. Both groups had higher \%Lag0 in the hour before sleep than in the hour after waking up, but the differences were not significant.

Table 2

Age dependencies of \%Lag0 in the hour before and after sleep among younger and older participants.

\begin{tabular}{|c|c|c|c|}
\hline & & Younger & Older \\
\hline \multirow[t]{4}{*}{ Hour before sleep (evening) } & \%lag0 between & $58.2 \pm 5.8$ & $30.7 \pm 5.1^{\star *}$ \\
\hline & PA and HFnu & & \\
\hline & \%lag0 between & $43.3 \pm 4.9$ & $18.3 \pm 3.9 * *$ \\
\hline & $\mathrm{PA}$ and $\mathrm{LF} / \mathrm{HF}$ & & \\
\hline \multirow[t]{4}{*}{ Hour after sleep (morning) } & \%lag0 between & $52.4 \pm 5.4$ & $24.5 \pm 4.8^{\star \star \star}$ \\
\hline & PA and HFnu & & \\
\hline & \%lag0 between & $31.1 \pm 4.5$ & $11.0 \pm 3.1^{*}$ \\
\hline & $\mathrm{PA}$ and $\mathrm{LF} / \mathrm{HF}$ & & \\
\hline
\end{tabular}

In Fig. 4, \%Lag0 between the time series of HFnu or LF/HF and of PA in the hour before sleep and after sleep are shown as low distress vs. high distress among the younger and older groups, respectively. In the hour before sleep, the older group with high scores of GHQ total and GHQ A-D had significantly lower \%Lag0 than the younger group. In the older group, participants with high scores of GHQ D (severe depression) both in HFnu and LF/HF showed significantly lower \%Lag0 than those with low scores. In the younger group, participants with high scores of GHQ D (severe depression) both in HFnu and LF/HF had lower \%lag0 than those with low scores; however, this was not significant.

In the hour after waking up, the older group with high scores of GHQ A-D (except vs. HFnu in B and C) had significantly lower \%Lag0 than the younger group. However, no significant difference was observed between the older groups with high GHQ D and the older groups with low GHQ D (severe depression), both in HFnu and LF/HF.

\section{Discussion}


In adult women, we confirmed that \%Lag0 is reduced with aging. It was significantly lower in older subjects before and after sleep than in younger subjects, as reported in our previous study [13]. Furthermore, older women with depression had significantly lower \%Lag0 in the hour before sleep. These results indicate that depression is closely associated with impairments in the coordination between HRV and PA before sleep in older women.

Psychological stress can be accurately evaluated using the total score of GHQ28 [28]. These findings appear to support our present results in older and younger individuals with depressive tendencies based on their GHQ28 subscale D scores and lower \%Lag0 between HRV and PA; however, the decrease was not significant in younger individuals. These results indicate that depression is closely associated with discoordination between HRV and PA only in older women, particularly in the hour before sleep. To the best of our knowledge, this study is the first to focus on the association of depression with coordination between HRV and PA in women.

By analysing \%Lag0, this study suggested that mental health conditions are closely associated with the coordination between HRV and PA in older women. The hypothalamic pituitary adrenal axis plays a significant role in controlling neuroendocrine stress responses [29]. In vivo human imaging studies reported that hypothalamic pituitary adrenal axis responses to a number of different stressful stimuli are gated through various brain regions [30,31]. All activities in the body that are initiated from the telencephalon, including those controlled by neuronal programs in the hypothalamus and mesencephalon, co-opt the lower brain stem centres [32]. PA is considered to be linked with the autonomic nervous system [33]. Therefore, impairments in the coordination between the HRV and PA may be derived from compromised links among their systems.

Patients with depression have been reported to have sleep disorders [34]. Participants with sleep difficulties are threefold to fourfold more likely to be depressed, and those with depression have low sleep efficiency [35]. In this study, no significant difference in \%Lag0 was detected between participants with or without incidents of nocturnal awakening on the experimental day or irregular sleep cycles, based on the questionnaires (data for reviewers). In the groups with depression, a close relationship was observed between HRV and depression before and during sleep, reflecting the coordination between HRV and PA.

The menopausal years in Japanese women reportedly begin at approximately 52 years of age. We divided subjects into 2 groups, namely, the younger group (59 years or younger) and the older group (60 years or older). Therefore, the older group might comprise only postmenopausal women. In contrast, some of the subjects in the younger group might also have been postmenopausal, as they were in their late forties and early fifties. Estrogen has been reported to reduce cardiomyocyte contractile function and sympathovagal nervous activity, whereas androgen enhances these [36]. Estrogen acts on the central nervous system to reduce sympathovagal activity [37]. In postmenopausal women, sympathovagal nervous activity is elevated due to changes in the hormonal balance [38]. Menopause or the menstruation cycle could also affect depression or distress [39]. 
Depression is strongly associated with physical disability [40], moreover, the prevalence of depression in patients with cardiac disease is quite variable [41]. We consider that our new index could help understand the connection between physical function and depression, and thus reduce cardiovascular diseases.

\section{Limitations}

The analytical method used in this study to evaluate the coordination between the HRV and PA has several limitations. We defined the beginning and end time points of a night's sleep based on changes in body position and the records of participants. Therefore, accurate identification of falling asleep, nocturnal waking, and waking up was not possible. In addition, as discussed above, we analysed the coordination between HRV and PA in the hour before sleep. We initially considered the higher sensitivity of \%Lag0 in the hour before sleep to be derived from equal activity patterns with fewer variations in PA. However, no significant differences were observed among PA, average PA, or coefficients of variation during the waking periods, even in the hour before and after sleep (data for reviewers). Physiological reasons for why the hour before sleep is the key time to examine the relationship between \%Lag0 and psychological distress remain unclear. In the present study, we could not establish whether menopause affected the \%Lag0 in younger and older women.

This study did not control for the comorbidity score or physical condition of participants. All participants had no or mild diseases. However, we considered that various kinds of metabolic and/or neuromuscular diseases could be involved in the discoordination. As the number of participants was limited, the effects of diseases on \%Lag0 remained unclear. Taking the above limitations into consideration, further controlled studies are required in the future.

\section{Conclusions}

By using the "\%Lag0" index, which was determined by the time-series correlation analysis between HRV and PA, the present study suggests that impairments in the coordination between HRV and PA are closely associated with depression in older women, particularly in the hour before sleep on free-moving days. This index may be able to detect depression using a non-invasive and quantitative analysis obtained from PA and HRV monitors in a clinical, or even home, settings.

\section{Abbreviation}




\begin{tabular}{|ll|}
\hline HRV & heart rate variability \\
\hline HF & high frequency \\
\hline LF & low frequency \\
\hline PA & physical acceleration \\
\hline GHQ28 & General Health Questionnaire \\
\hline$\%$ Lag0 & percent ratio of the lag $=0$ min in $1 \mathrm{~h}$ \\
\hline ECG & electrocardiogram \\
\hline
\end{tabular}

\section{Declarations}

\section{Ethics approval and consent to participate}

This study was approved by the Ethics Committee at the National Cerebral and Cardiovascular Research Center (M18-19-2, M26-158), Chubu University (280031) and Kyoto University (R1758). All participants provided written informed consents prior to participation in this study.

\section{Consent for publication}

All participants provided written and explicit consent for their anonymised data to be used in publications.

\section{Availability of data and materials}

The data generated during the current study will be available from the corresponding author on reasonable request and in accordance with consent and ethical approval.

\section{Competing interests}

The authors declare that they have no competing interests.

\section{Funding}

This study was supported in part by Grants-in-Aid from the Japanese Ministry of Education, Science, and Culture (Grants 17659207 to A. Shimouchi, and 15J08579 and 19K21435 to K. Taniguchi). The Center of Innovation, Science and Technology-based Radical Innovation and Entrepreneurship Program, Japan to A. Shimouchi. The Japan Agency for Medical Research and Development to A Shimouchi. The Intramural Research Fund of the National Cerebral and Cardiovascular Research Center (25-2-1) to A. Shimouchi. The funders had no role in study design, data collection and analysis, decision to publish, or preparation of the manuscript. 
KT performed all parts of the method and wrote all of the manuscript. A Shimouchi performed experimental plan and methodological procedures, rewrote all parts of the manuscript. NJ performed several parts of the experiment and gave advices for presentation. A Seiyama gave an idea of analysis and rewrote the original manuscript. All authors have read and approved the manuscript

\section{Acknowledgements}

We thank all the volunteers who participated in this study. We also would like to express special thanks to Ms. Noriko Inui, Mariko Mori, Azusa Kawamura, Shoko Nagahiro, Mariko Komatsu, Nobue Nishi, Hiroko Hayashi, and Yoshiko Kokusho for their technical assistance.

We presented a part of these results at the 94th Annual Meeting of the Physiological Society of Japan, 2017 (poster presentation).

\section{References}

1. Task Force of the European Society of Cardiology and the North American Society of Pacing and Electrophysiology. Heart rate variability: standards of measurement, physiological interpretation, and clinical use. Eur Heart J. 1996;93:1043-65.

2. Klieger RE, Bigger JT, Bosner MS. Stability over time of variables measuring heart rate variability in normal subjects. Am J Cardiol. 1992;68:626-30.

3. Stein C, Dal Lago P, Ferreira JB, Casali KR, Plentz RD. Transcutaneous electrical nerve stimulation at different frequencies on heart rate variability in healthy subjects. Auton Neurosci. 2011;165(2):2058.

4. Malliani A, Montano N. Heart rate variability as a clinical tool. Ital Heart J. 2002;3(8):439-45.

5. Ashare RL, Sinha R, Lampert R, Weinberger AH, Anderson GM, et al. Blunted vagal reactivity predicts stress-precipitated tobacco smoking. Psychopharmacology. 2012;220(2):259-68.

6. Pagani M, Lombardi F, Guzzetti S, Sandrone G, Rimoldi O, et al. Initial blood pressure fall on stand up and exercise explained by changes in total peripheral resistance. J Appl Physiol. 1991;70:523-30.

7. Eckberg DL. Sympathovagal balance: a critical appraisal. Circulation. 1997;96(9):3224-32.

8. Dubbert PM, White JD, Grothe KB, O'Jile J, Kirchner KA. Physical activity in patients who are severely mentally ill: feasibility of assessment for clinical and research applications. Arch Psychiatr Nurs. 2006;20:205-9.

9. Bauman A, Merom D, Bull FC, Buchner DM, Fiatarone SMA. Updating the evidence for physical activity: summative reviews of the epidemiological evidence, prevalence, and interventions to promote "active aging". Gerontologist. 2016;2:268-80.

10. Proper KI, Staal BJ, Hildebrandt VH, van der Beek AJ, van Mechelen W. Effectiveness of physical activity programs at worksites with respect to work-related outcomes. Scand J Work Environ Health. 2002;28(2):75-84. 
11. Rowell LB, O'Leary DS. Reflex control of the circulation during exercise: chemoreflexes and mechanoreflexes. J Appl Physiol (1985). 1990;69(2):407-18.

12. Abdullah H, Maddage NC, Cosic I, Cvetkovic D. Cross-correlation of EEG frequency bands and heart rate variability for sleep apnoea classification. Med Biol Eng Comput. 2010;48(12):1261-69.

13. Taniguchi K, Shimouchi A, Seki J, Jinno N, Shirai M, et al. Factors affecting coordination between heart rate variability and physical acceleration in daily lives of free-moving adults. Adv Biomed Eng. 2015;4:35-41.

14. Loprinzi PD, Herod SM, Cardinal BJ, Noakes TD. Physical activity and the brain: a review of this dynamic, bi-directional relationship. Brain Res. 2013;1539:95-104.

15. Craft LL, Perna FM. The benefits of exercise for the clinically depressed. Prim Care Companion J Clin Psychiatry. 2004;6:104-11.

16. McHenry J, Carrier N, Hull E, Kabbaj M. Sex differences in anxiety and depression: role of testosterone. Front Neuroendocrinol. 2014;35(1):42-57.

17. Aoyagi Y, Shephard RJ. Sex differences in relationships between habitual physical activity and health in the elderly: practical implications for epidemiologists based on pedometer/accelerometer data from the Nakanojo Study. Arch Gerontol Geriatr. 2013;56:327-38.

18. Koenig J, Thayer JF. Sex differences in healthy human heart rate variability: A meta-analysis. Neurosci Biobehav Rev. 2016;64:288-310.

19. Burst C, Wieling W, Van Brederode JFM, Hond A, De Rijk LG, et al. Mechanisms of initial heart rate response to postural change. Am J Physiol. 1982;243:H676-81.

20. Goldberg DP, Hillier VF. A scaled version of the General Health Questionnaire. Psychol Med 1979;139-145.

21. Iwata N, Saito K. The factor structure of the 28-item General Health Questionnaire when used in Japanese early adolescents and adult employees: age- and cross-cultural comparisons. Clin Neurosci. 1992;242(2-3):172-8.

22. Esperer HD, Esperer C, Cohen RJ. Cardiac arrhythmias imprint specific signatures on Lorenz plots. Ann Noninvasive Electrocardiol. 2008;13:44-60.

23. Taniguchi K, Shimouchi A, Jinno N, Okumura N, Seiyama A. Parasympathetic nervous activity associated with discoordination between physical acceleration and heart rate variability in patients with sleep apnea. Adv Exp Med Biol. in press.

24. Fukuda K, Kobayashi S. The Japanese version of the self-rating depression scale. Sankyoubo in Japanese 1983.

25. Iwashita S, Takeno Y, Okazaki K, Itoh J, Kamijo Y, et al. Triaxial accelerometry to evaluate walking efficiency in older subjects. Med Sci Sports Exec. 2003;35:1766-72.

26. Kato H, Asukai N, Miyake Y, Minakawa K, Nishiyama A. Post-traumatic symptoms among younger and elderly evacuees in the early stages following the 1995 Hanshin-Awaji earthquake in Japan. Acta Psychiatr Scand. 1996;93(6):477-81. 
27. Takanishi T, Ebara T, Murasaki Gl, Kubo T, Tachi N, et al. Interactive Model of Subsidiary Behaviors, Work Performance and Autonomic Nerve Activity during Working Hours. J Occup Health. 2010;52:39-47.

28. Goodwin L, Ben-Zion I, Fear NT, Hotopf M, Stansfeld SA, et al. Are reports of psychological stress higher in occupational studies? A systematic review across occupational and population based studies. PLoS One. 2013. 10.1371/journal.pone.0078693.

29. Jankord R, Herman JP. Limbic regulation of hypothalamo-pituitary-adrenocortical function during acute and chronic stress. Ann N Y Acad Sci. 2008;1148:64-73.

30. Holsen LM, Lee JH, Spaeth SB, Ogden LA, Klibanski A, et al. Brain hypoactivation, autonomic nervous system dysregulation, and gonadal hormones in depression: a preliminary study. Neurosci Lett. 2012;514:57-61.

31. Tobet SA, Handa RJ, Goldstein JM. Sex-dependent pathophysiology as predictors of comorbidity of major depressive disorder and cardiovascular disease. Pflugers Arch. 2013;465:585-94.

32. Janig W. Integrative action of the autonomic nervous system Cambridge University Press 2006:459516.

33. Mueller PJ. Exercise training and sympathetic nervous system activity: evidence for physical activity dependent neural plasticity. Clin Exp Pharmacol Physiol. 2007;34:377-84.

34. Franzen PL, Buysse DJ. Sleep disturbances and depression: risk relationships for subsequent depression and therapeutic implications. Dialogues Clin Neurosci. 2008;10:473-81.

35. Almeida OP, Pfaff JJ. Sleep complaints among older general practice patients: association with depression. Br J Gen Pract. 2005;55:864-6.

36. Gold EB, Bromberger J, Crawford S, Samuels S, Greendale GA, et al. Factors associated with age at natural menopause in a multiethnic sample of midlife women. Am J Epidemiol. 2001;153:865-74.

37. Joyner MJ, Barnes JN, Hart EC, Wallin BC, Charkoudian N. Neural control of the circulation: how sex and age differences interact in humans. Compr Physiol. 2015;5:193-215.

38. Magri F, Gabellieri E, Busconi L, Guazzoni V, Cravello L, et al. Cardiovascular, anthropometric and neurocognitive features of healthy postmenopausal women: effects of hormone replacement therapy. Life Sci. 2006;78:2625-32.

39. Payne JL, Roy PS, Murphy-Eberenz K, Weismann MM, Swartz KL, et al. Reproductive cycle-associated mood symptoms in women with major depression and bipolar disorder. J Affect Disord. 2007;99:221-9.

40. Djernes JK. Prevalence and predictors of depression in populations of elderly: a review. Acta Psychiatr Scand. 2006;113(5):372-87.

41. Hare DL, Toukhsati SR, Johansson P, Jaarsma T. Depression and cardiovascular disease: a clinical review. Eur Heart J. 2014;35(21):1365-72.

\section{Figures}


106 women participated in this study

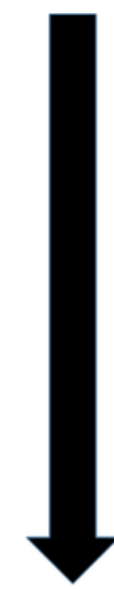

11 subjects were excluded

-6 with severe arrhythmia

-4 drank alcohol on the experiment day

-3 with excessive electrical noise in the devices

-2 used beta-blocker

(numbers are overlapped)

95 women analyzed in this study

Figure 1

Study population for the evaluation of the coordination between HRV and PA. 

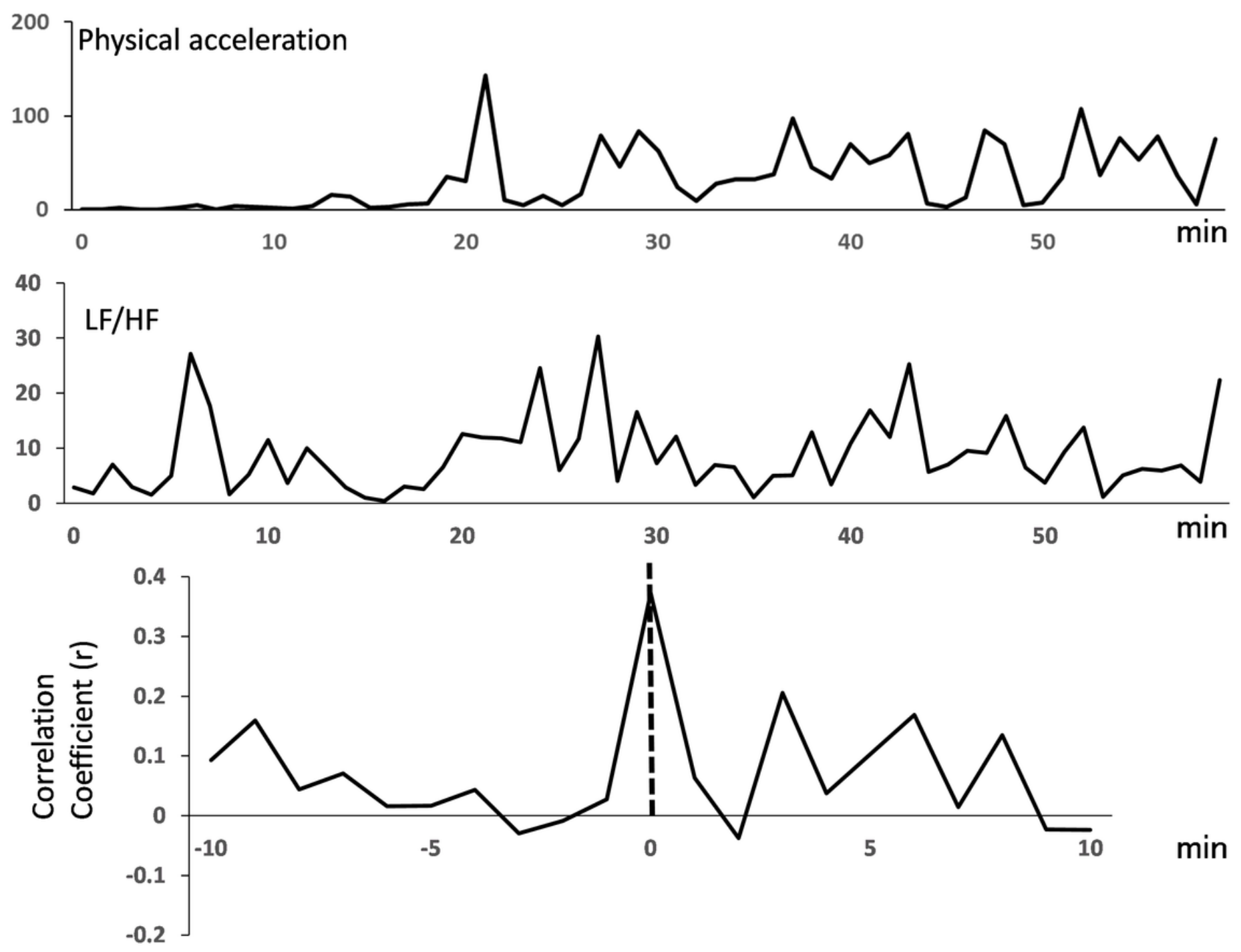

Figure 2

$\mathrm{Lag}=0$ of PA and LF/HF 

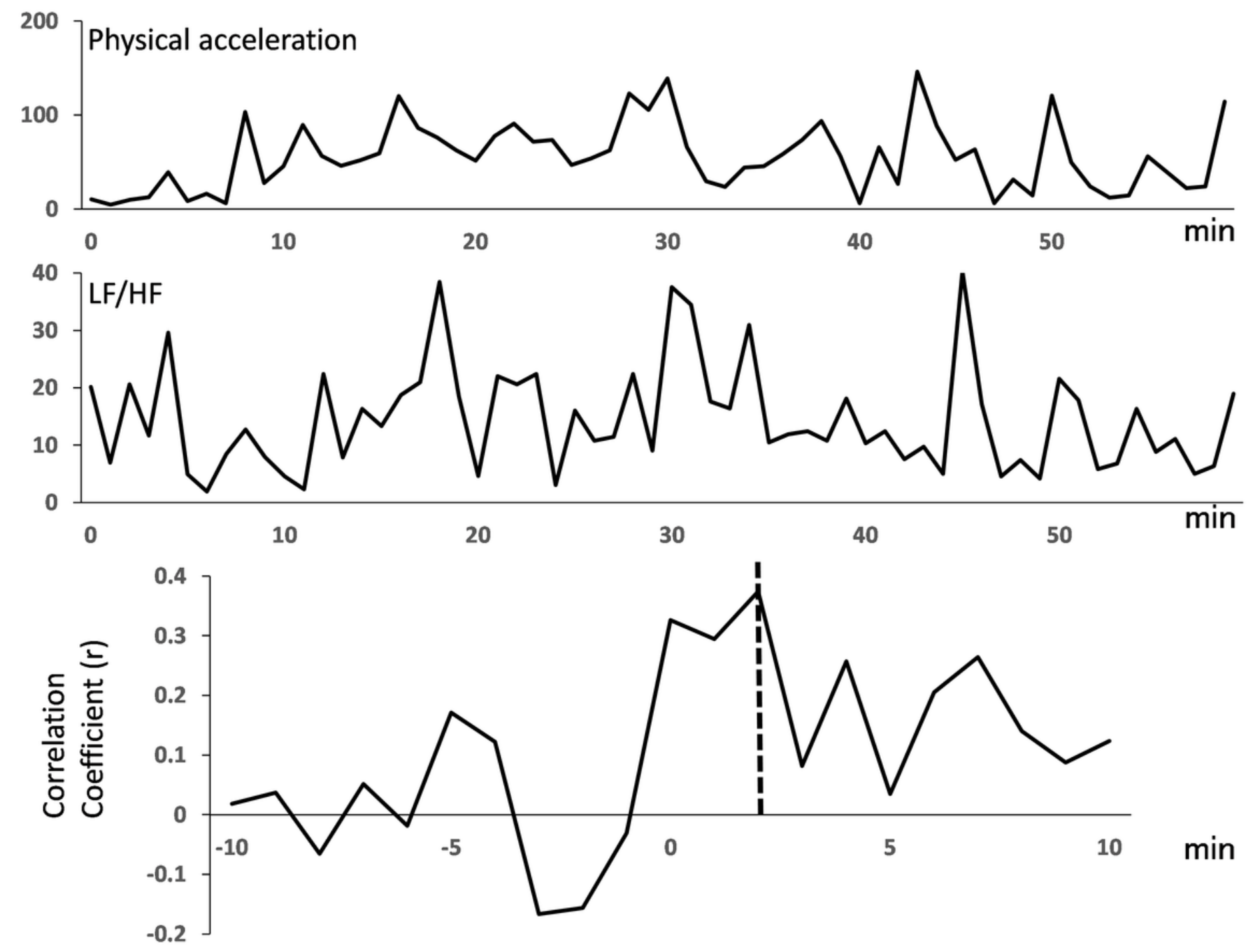

Figure 3

Lag $=2$ min of PA and LF/HF before night sleep 


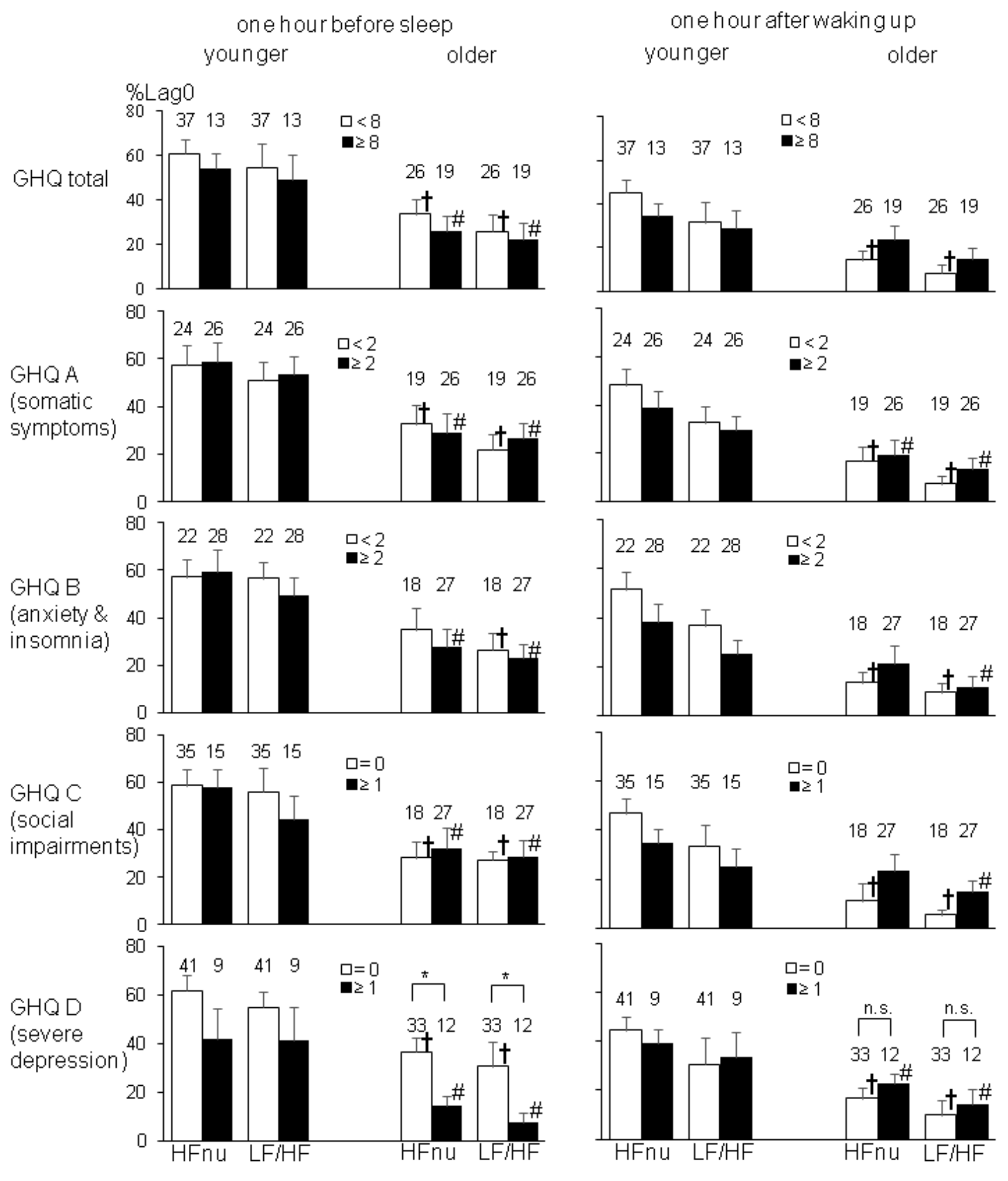

Figure 4

Relationships between \%Lag0 and GHQ scores in the younger and older groups. Values above error bars show the number of subjects. + : $p<0.05$, younger vs. older subjects with low score of GHQ; \#: $p<0.05$, younger vs. older subjects with high score of GHQ; *: $p<0.05$, older subjects with low score of GHQ D vs. older subjects with high score of GHQ D. Error bars represent standard error. 


\section{Supplementary Files}

This is a list of supplementary files associated with this preprint. Click to download.

- QuestionnireinEnglishGHQ28.pdf

- QuestionnaireinJapaneseGHQ28.pdf

- supplementaldata.pdf 